\title{
Probing the Heme Reactive Center of Dehaloperoxidase from Amphitrite ornata via Spectroscopic, X-ray and Neutron Crystallographic Methods
}

\author{
Leiah Carey and Reza Ghiladi \\ North Carolina State University
}

The relationship between substrate binding and enzymatic function has been well established in monofunctional enzymatic systems, however complete understanding in multifunctional systems is still lacking. Our chosen platform for the elaboration of the structural features and other determinants that impart specific discrete functions to heme proteins (and more generally to all proteins) is the enzyme dehaloperoxidase (DHP), the coelomic hemoglobin from the marine worm Amphitrite ornata. DHP is the first globin to possess biologically relevant peroxidase activity, and in the past 3 years peroxygenase, oxidase, and oxygenase activities were discovered in the Ghiladi Lab at NCSU. Thus, 5 functionalities have been discovered to coexist on the single heme active site of DHP. In an attempt to explain this functional coexistance, an investigation into the structural consequence on function is presented here. In particular, crystallographic and spectroscopic analysis of substrate binding is discussed in regard to their respective functions. In an effort to probe more subtle structural details, the first neutron diffraction structure of a multi-functional heme protein is presented and analyzed against monofunctional peroxidase and globin neutron structures. 